ORIGINAL PAPER

\title{
ROLE OF MAST CELLS IN THE PATHOGENESIS OF LIVER FIBROSIS IN NONALCOHOLIC FATTY LIVER DISEASE
}

\author{
Ewa A. Lewandowska ${ }^{1}$, Agnieszka Wosiak ${ }^{2}$, Andrzej Zieliński ${ }^{1}$, Piotr Brzeziński ${ }^{1}$, \\ Janusz StrzelczyK ${ }^{3}$, Dariusz Szymański ${ }^{3}$, Józef Kobos ${ }^{1}$
}

\author{
${ }^{1}$ Department of Histology and Embryology, Interfaculty Chair of Anatomy and Histology, Medical University of Lodz, \\ Lodz, Poland \\ ${ }^{2}$ Information Technology Institute, Technical University of Lodz, Lodz, Poland \\ ${ }^{3}$ Department of General and Transplant Surgery, Norbert Barlicki Memorial Teaching Hospital No. 1, Medical \\ University of Lodz, Lodz, Poland
}

\begin{abstract}
Nonalcoholic fatty liver disease (NAFLD), a very common comorbidity of obesity, may progress from simple steatosis to nonalcoholic steatohepatitis and liver cirrhosis. The aim of this study was to determine whether mast cells (MCs) participate in the pathogenesis of liver fibrosis in patients with NAFLD.

Liver specimens collected either from obese subjects or the control group were prepared for histological examination. The patients were divided into groups depending on inflammation and fibrosis grade, and the mean total number of mast cells $/ \mathrm{mm}^{2}$ and those located only in portal areas/ fibrous septa or within lobules were calculated for each grade. Mast cells were detected by pina-cyanol erythrosinate staining.

There was a strong positive correlation between the number of mast cells, especially those located within portal areas and fibrous septa, and the liver fibrosis grade $(\mathrm{r}=0.736, \mathrm{p}<0.0001)$. No similar dependency between the number of mastocytes within lobules and liver fibrosis grade was identified.

The results from this study suggest the involvement of mast cells from portal areas and fibrous septa in the pathogenesis of liver fibrosis in patients with NAFLD.
\end{abstract}

Key words: mast cells, liver fibrosis, NAFLD, obesity.

\section{Introduction}

Obesity, especially its abdominal type, carries an increased risk of many comorbidities including nonalcoholic fatty liver disease. NAFLD affects approx. $75 \%$ of obese people and comprises subtypes such as simple steatosis, nonalcoholic steatohepatitis (NASH), and cirrhosis, which can evolve to hepatocellular carcinoma (HCC) [1]. Liver cirrhosis is a late stage of organ fibrosis characterised by the presence of regenerative nodules surrounded by fibrous septa that distort the natural structure of the liver $[2,3,4,5]$. These changes in liver histology lead to portal hypertension and organ failure [5]. In the United States, NASH is the third leading cause of liver cirrhosis, after alcohol abuse and HCV infection [6]. Due to both a high incidence of NAFLD in the world and the risk of its progression to cirrhosis, it is imperative to look for causes of the aforementioned phenomenon in order to implement an effective anti-fibrotic therapy.

It is an established fact that fibrosis results from an imbalance between deposition and degradation of extracellular matrix (ECM) [7]. Some ECM proteins 
that are secreted in excessive amounts feature type I and IV collagen as well as fibronectin, elastin, and laminin [8]. Hepatic stellate cells (HSCs) play a major role in the production of extracellular matrix constituents in the liver and are activated by an inflammation process, resulting from damage to hepatic parenchyma. Once activated, HSCs transform into cells that resemble myofibroblasts in terms of phenotype and are responsible for an increased protein deposition [9]. Moreover, it has been shown that activated HSCs release factors that reduce the degradation of extracellular matrix, which, in turn, further impairs the balance between the synthesis and degradation of matrix components. Apart from hepatic stellate cells, also portal fibroblasts and bone marrow-derived myofibroblasts show profibrogenic properties in the damaged liver $[10,11]$. The activation of cells that stimulate fibrogenesis predominantly occurs by means of the transforming growth factor $\beta$ (TGF- $\beta$ ) secreted by inflammatory cells infiltrating the affected liver [9, 12].

Among cells that may be involved in the process of liver fibrosis are mast cells (mastocytes, MCs), which originate from haematopoietic progenitor cells [13]. Mastocytes contain many mediators and cytokines in their cytoplasmic granules such as TGF- $\beta$, which is responsible for hepatic stellate cell activation. Mastocyte degranulation affects their environment due to the induction of inflammation and the attraction of other inflammatory cells $[14]$. Several studies indicate the role of mast cells in the fibrosis of various organs $[15,16,17,18,19,20,21]$. On the one hand, numerous studies have shown that the number of mast cells in portal areas and fibrous septa increases in late stages of liver fibrosis [22, $23,24,25]$. On the other hand, research on mast cell-deficient rodent models suggest that MCs do not play a central role in the pathogenesis of the fibrosis of the organ in question [26].

\section{Aim of the study}

Only limited data are available in the literature as regards the interdependence between the number of mast cells and the advancement stage of liver fibrosis and inflammation in nonalcoholic fatty liver disease. Therefore, it is essential to examine whether the progression of fibrosis is associated with an increased number of MCs and/or with intensified inflammation in the liver parenchyma in patients with NAFLD. The aim of this study was to determine whether the MCs participate in the pathogenesis of liver fibrosis in patients with NAFLD, and which of the mastocytes have a greater impact on the process: cells located in the portal areas/fibrous septa or those within the liver lobules. It was also interesting for us to investigate which of the two following factors affects the development of liver fibrosis to a greater degree: mast cell content or stage of inflammation (portal and intralobular). The study complies with up-to-date global standards of histological scoring and grading systems approved for NAFLD.

\section{Material and methods}

The study material comprised liver specimens collected from obese subjects who underwent bariatric surgery in Norbert Barlicki Memorial Teaching Hospital No. 1 of the Medical University of Lodz. Indication for surgery was class III obesity (BMI > 40) or class II obesity (BMI 35-40) with obesity-related complications (such as type II diabetes, hypertension, or lipid disorders) and insufficient weight loss despite the therapy undertaken.

Forty-seven patients with features of NAFLD were enrolled in the study ( 32 females and 15 males). The average BMI in the study group was $45.8 \pm 5.84$, and the mean age of the patients was 41 years. The control material was collected during routine autopsies (without signs of lesions to the organs), carried out at the Department of Forensic Medicine of the Medical University of Lodz. The exclusion criteria included: overweight, obesity, and alcohol poisoning as the cause of death since the latter implied alcoholism. Ten specimens of liver were included in the control group. All specimens were obtained from males. The average BMI in the control group was $21.5 \pm 2.9$, and the mean age amounted to 48 years.

\section{Methods}

\section{Staining and specimen assessment}

Immediately upon collection, tissues were fixed in $10 \%$ buffered formalin and prepared for histological examination. Sections were stained with haematoxylin and eosin, pina-cyanol erythrosinate [27], and Mallory trichrome stain.

Histological specimens were analysed by means of a computer image analysis system consisting of an Authentic ADM computer with an AMD Duron TM processor and a graphics processor combined with a Sony SSC-DC58AP video camera and a Carl Zeiss Jena Jenamed2 optical microscope. The specimens were assessed at various magnifications: $50 \times, 200 \times, 400 \times$, and $1000 \times$. The microscopic images were collected by a video camera that generated an analogue output signal. After the signal was converted from analogue to digital, it was transmitted for morphometric analysis. An analysis was performed using the program MultiScan Base v. 11.06 . 


\section{The number and location of mast cells in the organ}

Pina-cyanol erythrosinate (PE)-stained specimens were used for mast cell identification [27]. For each specimen, the mean number of mast cells $/ \mathrm{mm}^{2}$ was calculated based on the evaluation of 30 images magnified by 200'. Then the percentage of MCs located (a) in the portal areas and fibrous septa and (b) in the liver lobules was calculated. Mast cells were identified by virtue of their purple or violet-blue colour as a result of pina-cyanol erythrosinate staining as well as by virtue of the presence of cytoplasmic granules visible at large magnifications (400', 1000').

\section{Grade of portal inflammation, intralobular inflammation, and fibrosis}

The progress of portal and lobular inflammation was assessed at a magnification of 200', while 50', $200^{\prime}$, and 400' magnifications served for the evaluation of the advancement of fibrosis. For the assessment of each parameter, $\geq 30$ images were analysed. The results were classified in accordance with the standards set out in the Table I.

\section{Statistical methods}

The obtained results were analysed in statistical terms. Patients were divided into four groups depending on the fibrosis grade, in order to determine the mean number of mast cells $/ \mathrm{mm}^{2}$ for each grade and the mean number of $\mathrm{MCs} / \mathrm{mm}^{2}$ restricted to portal areas and fibrous septa or within lobules. One-factor analysis of variance (ANOVA) was used to test the differences. The relationships between following parameters: MC count, fibrosis grade, portal inflammation grade, and lobular inflammation grade, were determined using Spearman rank correlation coefficient. The level of statistical significance was set at $\mathrm{p} \leq 0.05$.

\section{Results}

\section{Fibrosis grade and content of $\mathrm{MCs} / \mathrm{mm}^{2}$}

A significantly higher mean number of $\mathrm{MCs} / \mathrm{mm}^{2}$ were found $\left(10.49 \pm 5.90 \mathrm{MCs} / \mathrm{mm}^{2}\right)$ in patients with features of NAFLD as opposed to the control group $\left(2.11 \pm 1.76 \mathrm{MCs} / \mathrm{mm}^{2} ; \mathrm{p}<0.0001\right)$.

The features of fibrosis were found in $93 \%$ of patients with NAFLD. In three cases no signs of fibrosis were noted in accordance with the Kleiner et al. scoring system [29]. Most patients showed fibrosis grade 3 and 2 (40.4\% and $31.9 \%$, respectively). Perisinusoidal and periportal fibrosis (grade 1) was observed in $21.3 \%$ of patients, whereas cirrhosis was not

Table I. List of criteria applied for the histological evaluation of liver inflammation and fibrosis

\begin{tabular}{|c|c|c|c|}
\hline ITEM & & SCORE AND DEFINITION & STAINING \\
\hline \multirow[t]{4}{*}{ Portal inflammation } & \multicolumn{2}{|r|}{ Scale of Brunt EM, Kleiner DE, Wilson LA, et al. [28] } & \multirow[t]{4}{*}{$\mathrm{HE}$} \\
\hline & 0 (none) & $\begin{array}{c}\text { No inflammation or only rare inflammatory cells in any } \\
\text { portal area; }\end{array}$ & \\
\hline & 1 (mild) & $\begin{array}{l}\text { A few mononuclear cells, usually, but not always, in more } \\
\text { than one portal tract; }\end{array}$ & \\
\hline & $\begin{array}{l}2 \text { (more } \\
\text { than mild) }\end{array}$ & $\begin{array}{c}\text { Moderate to marked density of inflammation in at } \\
\text { least one portal area, and/or the presence of lymphoid } \\
\text { aggregates. }\end{array}$ & \\
\hline \multirow[t]{5}{*}{ Lobular inflammation } & \multicolumn{2}{|c|}{ Scale of Kleiner DE, Brunt EM, Van Natta M, et al. [29] } & \multirow[t]{5}{*}{$\mathrm{HE}$} \\
\hline & 0 & No foci & \\
\hline & 1 & $<2$ foci & \\
\hline & 2 & $2-4$ foci & \\
\hline & 3 & $>4$ foci & \\
\hline \multirow[t]{6}{*}{ Liver fibrosis } & \multicolumn{2}{|c|}{ Scale of Kleiner DE, Brunt EM, Van Natta M, et al. [29] } & Mallory trichrome \\
\hline & 0 & None & \\
\hline & 1 & Perisinusoidal or periportal & \\
\hline & 2 & Perisinusoidal and portal/periportal & \\
\hline & 3 & Bridging fibrosis & \\
\hline & 4 & Cirrhosis & \\
\hline
\end{tabular}




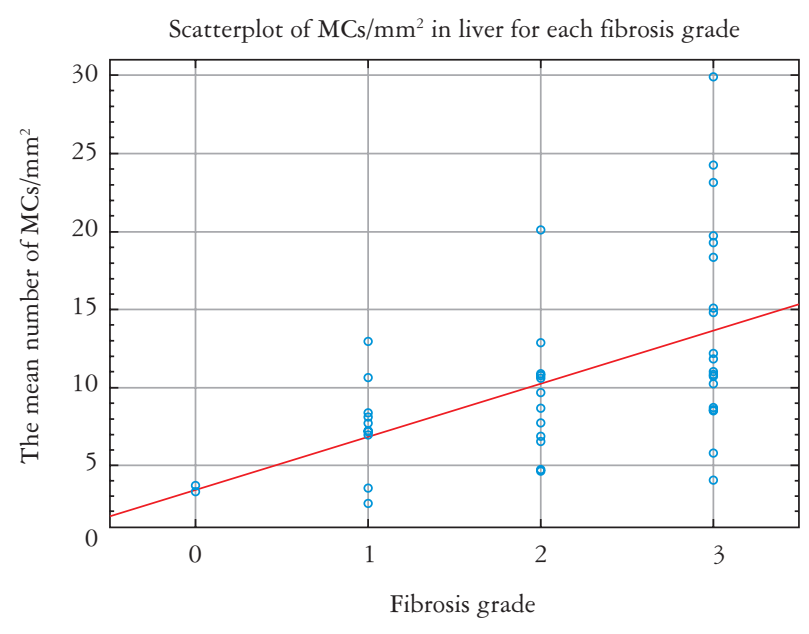

Fig. 1. Correlation between number of mast cells and fibrosis grade

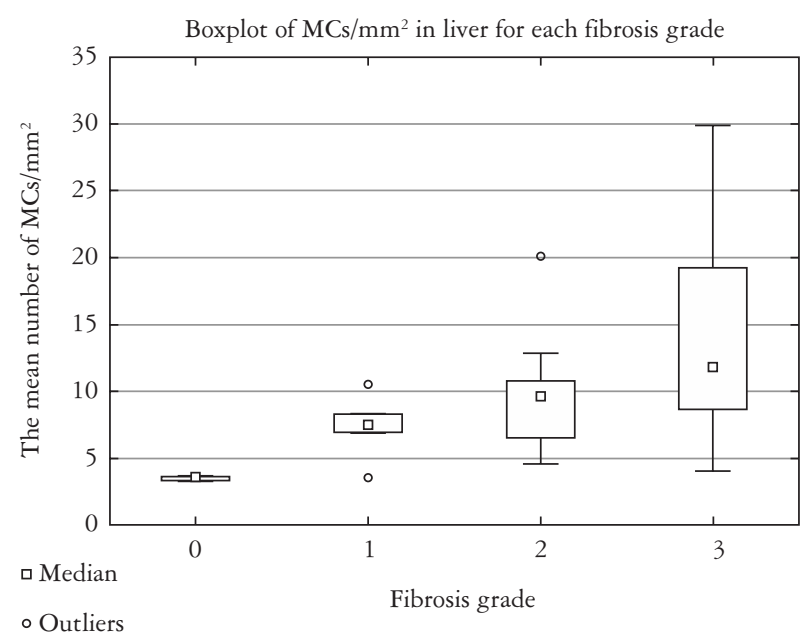

Fig. 2. The mean number of mast cells for each fibrosis grade

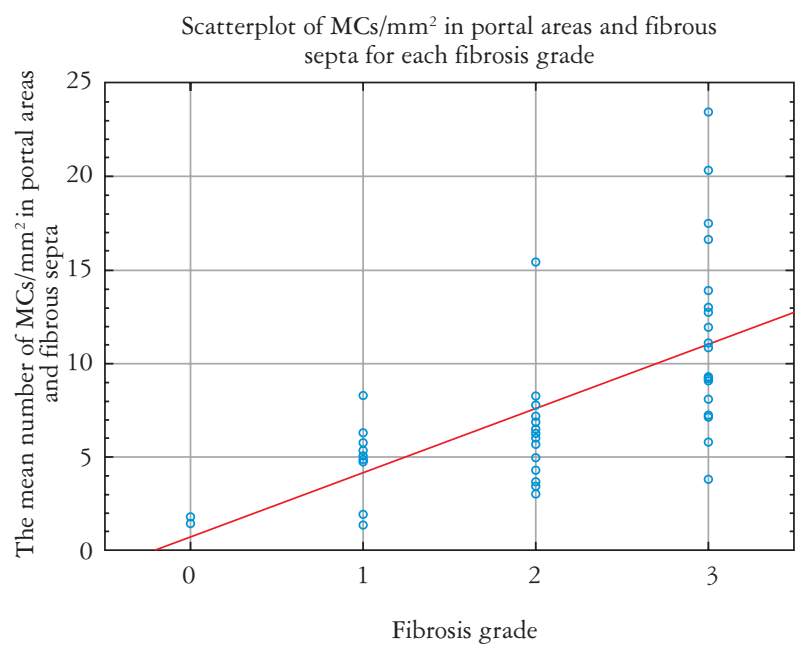

Fig. 3. Correlation between content of mast cells (restricted to portal areas and fibrous septa) and fibrosis grade detected in any of the cases under analysis (grade 4). No features indicative of fibrosis were found in the control group.

A significant positive correlation between the mean number of mast cells and the grade of fibrosis was found in the study group $(\mathrm{r}=0.585 ; \mathrm{p}<0.0001$, Fig. 1).

The average number of MCs in patients without signs of fibrosis reached $3.54 \pm 0.2 \mathrm{MCs} / \mathrm{mm}^{2}$. The mean number of MCs increased in the case of more advanced fibrosis and amounted to: 7.52 $\pm 2.99 \mathrm{MCs} / \mathrm{mm}^{2}$ (grade 1), $9.38 \pm 3.82 \mathrm{MCs} / \mathrm{mm}^{2}$ (grade 2), and $14.02 \pm 6.78 \mathrm{MCs} / \mathrm{mm}^{2}$ (grade 3), respectively (Fig. 2). The variations in MC content for the respective grades of fibrosis were statistically significant $(\mathrm{p}<0.001)$.

\section{Fibrosis grade vs. mast cell number in portal areas and fibrous septa}

In the study group, $74.2 \%$ of all identified mast cells were found within portal areas and fibrous septa. The study showed a strong positive correlation between fibrosis grade and the count of $\mathrm{MCs} / \mathrm{mm}^{2}$ restricted to portal area and fibrous septa $(\mathrm{r}=0.736$, $\mathrm{p}<0.0001$; Fig. 3).

The mean number of MCs in portal areas and fibrous septa for each fibrosis grade amounted to: 1.53 $\pm 0.22 \mathrm{MCs} / \mathrm{mm}^{2}$ (no fibrosis), $4.65 \pm 2.06 \mathrm{MCs} /$ $\mathrm{mm}^{2}$ (grade 1), $6.40 \pm 2.96 \mathrm{MCs} / \mathrm{mm}^{2}$ (grade 2), and $11.51 \pm 5.07 \mathrm{MCs} / \mathrm{mm}^{2}$ (grade 3), respectively. The variations in the mean number of MCs in portal areas and fibrous septa for the listed grades of fibrosis were statistically significant $(\mathrm{p}=0.00001)$.

\section{Fibrosis grade vs. mast cell number within liver} lobules

Approx. 25.8\% of all identified mast cells were found within liver lobules. No statistically significant correlation between fibrosis grade and $\mathrm{MCs} / \mathrm{mm}^{2}$ in liver lobules was found in patients with NAFLD features $(r=-0.096, p=0.519)$.

The average mast cell number in the above location varied for the respective fibrosis grades: $2.26 \pm 2.02$ $\mathrm{MCs} / \mathrm{mm}^{2}$ (no fibrosis), $2.87 \pm 1.28 \mathrm{MCs} / \mathrm{mm}^{2}$ (grade 1), $2.97 \pm 1.52 \mathrm{MCs} / \mathrm{mm}^{2}$ (grade 2), and $2.52 \pm 2.04$ $\mathrm{MCs} / \mathrm{mm}^{2}$ (grade 3). The variations in MCs content within lobules for various fibrosis grades were not statistically significant $(p=0.748)$.

\section{Fibrosis grade and portal inflammation grade}

No portal inflammation was observed in as few as $4.3 \%$ of patients. $29.8 \%$ of cases manifested grade 1 portal inflammation, whereas grade 2 was observed in $66 \%$ of patients. In the control group, $80 \%$ of cases showed no portal inflammation, with grade 1 inflammation present in $20 \%$ of cases. There was no 
case of grade 2 portal inflammation in the control group. The two groups differed significantly in terms of the above parameter $(p<0.05)$.

In patients with NAFLD, a statistically significant correlation between the grade of fibrosis and portal inflammation grade was found $(r=0.378$, $\mathrm{p}=0.009)$.

\section{Fibrosis grade and lobular inflammation grade}

Only a single subject in the study was found to be free from lobular inflammation. Most cases indicated grade $1(42.6 \%)$ and $2(31.9 \%)$ lobular inflammation, whereas grade 3 was detected in $23.4 \%$ of patients. All subjects in the control group suffered from grade 1 lobular inflammation. The differences between groups were statistically significant $(\mathrm{p}<0.05)$.

In patients with NAFLD, there was no statistically significant correlation between fibrosis grade and lobular inflammation grade $(r=0.197, \mathrm{p}=0.183)$.

\section{Mast cells number vs. advancement stage of liver inflammation}

The study demonstrated a significant positive correlation between $\mathrm{MCs} / \mathrm{mm}^{2}$ and portal inflammation grade $(\mathrm{r}=0.389, \mathrm{p}=0.007)$ as well as between $\mathrm{MCs} / \mathrm{mm}^{2}$ of portal areas and fibrous septa, on the one hand, and portal inflammation grade, on the other $(r=0.404, p=0.005)$. No such interdependencies occurred between $\mathrm{MCs} / \mathrm{mm}^{2}$ and lobular inflammation grade $(\mathrm{r}=0.00086, \mathrm{p}=0.995)$ or between $\mathrm{MCs} / \mathrm{mm}^{2}$ within the lobules and lobular inflammation grade $(\mathrm{r}=-0.090, \mathrm{p}=0.548)$.

\section{Discussion}

The pathogenesis of liver fibrosis in NASH patients has been poorly understood. In search of an explanation, a two-hit hypothesis has been put forth, according to which insulin resistance is a first step in liver steatosis. Insulin resistance that enhances fat tissue lipolysis and drives hyperinsulinism contributes to an increased influx of free fatty acid into the liver and the accumulation of triglycerides within hepatocytes [30]. The second hit comes with an initiation of inflammatory process and fibrosis in the liver, resulting from the combined impact of proinflammatory agents, oxidative stress, adipokines, and mitochondrial dysfunction [31]. Numerous studies have focused on seeking the causes of the progression from simple steatosis to nonalcoholic steatohepatitis due to the severity of its consequences, of which liver cirrhosis is the gravest. MCs have been suggested to participate in the initiation of liver inflammation and fibrosis [25, 32].

Human liver contains a relatively low number of mast cells: from 1.2 to $3.9 \mathrm{MCs} / \mathrm{mm}^{2}$ [33]. This

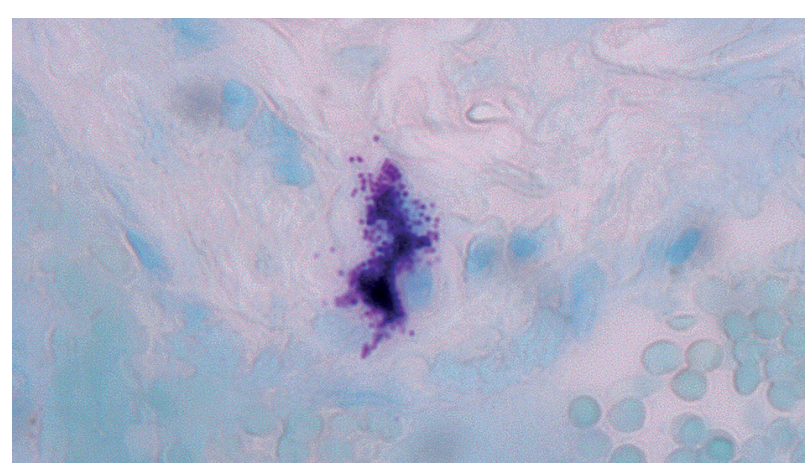

Fig. 4. Purple granules of degranulating mast cell are clearly visible at high magnification $(1000 \times)$. Pina-cyanol erythrosinate staining

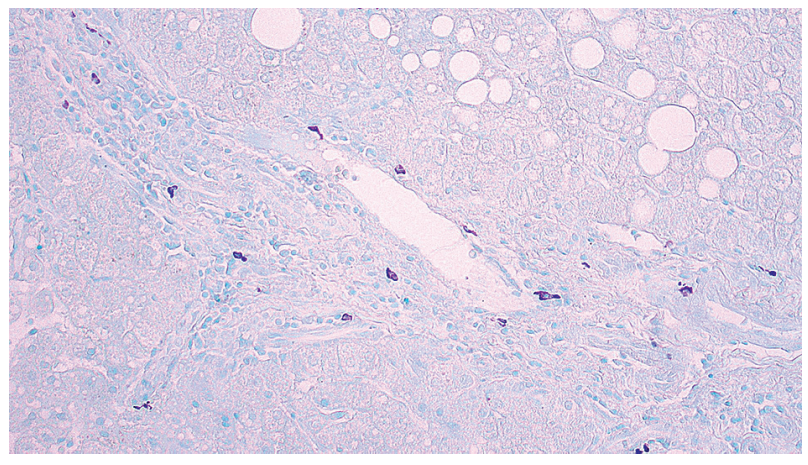

Fig. 5. Accumulation of mast cells in the portal tract. Magnification $200 \times$. Pina-cyanol erythrosinate staining

hypothesis that MCs play a role in liver fibrosis may be corroborated by the fact that their count rises in the liver affected by late stages of fibrosis, including cirrhosis [22, 23, 24, 25]. Mastocytes might participate in the pathogenesis of fibrosis by releasing mediators from the granules. These mediators include TGF- $\beta$, which stimulates hepatic stellate cells to produce ECM components, as well as tryptase, which promotes collagen synthesis by fibroblasts and fibroblast growth $[34,35]$. Other factors contained in MC granules that induce fibrosis feature PDFG (platelet derived growth factor) and GM-CSF (granulocyte macrophage colony stimulating factor [36], histamine, heparin, and bFGF [basis fibroblast growth factor]) [25]. In addition, mast cells are also a source of inflammatory factors such as TNF- $\alpha$ (tumour necrosis factor- $\alpha$ ), which activates many cells and initiates the inflammatory process [14]. Further still, mast cells support the profibrogenic response of Th-2 due to the content of factors such as IL- 4 or IL-10.

The study showed that MC count in patients with NAFLD increases relative to fibrosis stage, as assessed according to the NASH Clinical Research Network Scoring System Definitions and Scores [29]. This interdependence proves to be even stronger for mast cells restricted to portal areas and fibrous septa. This, in turn, may suggest that mastocytes directly affect 
fibrosis progression in patients with NAFLD. The higher accumulation of MCs in portal tracts and fibrous septa might be related to an increased number of microvessels in those locations, specific to chronic liver damage $[37,38]$. Mastocytes are believed to have angiogenic potential. Mast cells under both physiological and pathophysiological conditions accumulate in the close proximity of capillaries, including locations such as tumour growth, wound healing, and tissue repair - the places where angiogenesis is prominent $[39,40,41]$. MCs are also a source of angiogenic mediators such as: fibroblast growth factor (FGF)-2, vascular endothelial growth factor (VEGF) [42], tryptase, chymase [13], histamine [43], heparin [44], and matrix metalloproteinase-9 (MMP-9) [45]. The abovementioned factors regulate the endothelial cell proliferation and function and/or degrade ECM components to provide space for neovascular growth. Recent studies have demonstrated that angiogenesis might contribute to the liver fibrosis progression during the process of wound healing in chronic liver diseases [38, 46, 47, 48].

Moreover, our study demonstrated that the mean number of mast cells in liver with NAFLD features is significantly higher than in normal human liver. A similar observation was made in a study conducted by Armbrust et al., which showed an increase in the number of MCs in portal areas and fibrous septa in human and rat liver affected by cirrhosis, thereby suggesting MC participation in late stages of liver fibrosis. However, the latter study was limited to the analysis of MC count in two polar cases: no fibrosis or cirrhosis, disregarding intermediate stages of fibrosis [22]. In effect, the correlation between various stages of fibrosis and MC content was excluded in the cited study. Furthermore, this research concerned either artificially caused cirrhosis or cirrhosis derived from diseases other than NAFLD. A positive correlation between $\mathrm{MC}$ content and liver fibrosis was also demonstrated in other studies $[23,24,25]$, but none of them referred to NAFLD patients and none was assessed in compliance with NASH Clinical Research Network Scoring System Definitions and Scores.

Contrary to the above, Sugihara et al. put forward an alternative hypothesis based on the research of mast cell deficient rats $\mathrm{Ws} / \mathrm{Ws}$ and mice $\mathrm{W} / \mathrm{W}^{\mathrm{v}}$. In their study, liver cirrhosis was caused in the aforementioned rodents by the resection of bile duct or $\mathrm{CCl}_{4}$ induction. Sugihara contends that mast cells do not play a central role in the pathogenesis of liver fibrosis because cirrhosis occurred despite the lack of MCs in the animals studied [26].

It is an established fact that fibrogenesis requires the retention of signals attributed to chronic liver diseases caused by infection, medicines, metabolism disorders, etc., for activation. Although, occasionally, liver fibrosis might progress radically in a few weeks or months as a result of failure caused by hepatotoxins, HCV following transplantation [49], or concurrent HIV and HCV infections [50]; this process normally takes years [2]. In the study of Sugihara et al., liver cirrhosis was artificially provoked in the assessed rodents; for instance, by $\mathrm{CCl}_{4}$ induction resulting from acute organ injury. Cirrhosis advanced rapidly in the animals, and MC content was assessed relatively shortly after the causal factor occurred. Obesity and its comorbidities (such as liver fibrosis) develop over extended periods of time, and it is difficult to draw a comparison between the results obtained by us and the findings of Sugihara et al. The research of Charlton et al. may confirm that liver fibrosis in NASH subjects is a relatively long-term process. In the cited study, the features of progressive liver fibrosis in mice were not obtained sooner than after six months of a regular fast-food diet [51]. The study by Sugihara et al., wherein liver cirrhosis was observed in rodents despite the lack of MCs, does not rule out the existence of two different mechanisms underlying liver fibrosis in acute and chronic organ injury.

However, our research demonstrated that mast cell content within liver lobules does not increase with the progression of fibrosis, thereby inviting the suggestion that MCs in the above location do not play an important role in liver fibrosis in patients with NAFLD.

Our study showed that liver fibrosis grade in NAFLD patients increases proportionately to portal inflammation grade, as assessed in accordance with the Brunt et al. system [28]. However, no similar interdependence was identified between liver fibrosis grade and lobular inflammation grade. Chronic inflammatory process in the liver typically precedes the occurrence of fibrosis [2]. If the inflammation does not subside, fibrosis may evolve into more advanced stages, including liver cirrhosis [52, 53]. Liver fibrosis begins with the stimulation of the secretion of growth factors and cytokines by inflammatory cells, which activate the hepatic stellate cells to the synthesis of extracellular matrix components [2].

In a study concerning alcoholic liver disease (ALD), Colombat et al. demonstrated a positive correlation between portal inflammation and liver fibrosis. The authors suggested the role of portal inflammation in liver fibrosis among patients with ALD [54]. A positive interdependence between portal inflammation and late stages of liver fibrosis in obese patients was observed in studies by Liew et al. [55] and Brunt et al., among others. A total of 728 adults with NAFLD were enrolled in the study by Brunt $e t$ al. The authors indicated portal inflammation grade 1 and 2 as a marker of advanced disease [28]. The lack of correlation between lobular inflammation grade and fibrosis grade demonstrated in our study may suggest that inflammatory process within the liver 
lobules has no impact on fibrosis progression, contrary to inflammation within portal areas.

We also noted in our study a positive relationship between mast cell content and portal inflammation grade. Mast cells release many inflammatory factors, which, in turn, attract other inflammatory cells to the site of mast cell degranulation. This activates inflammatory cells and initiates the inflammatory process [14], which may cause liver fibrosis, if chronic $[2,52,53]$. The positive correlation between fibrosis grade and mast cell number, on the one hand, as well as between fibrosis grade and portal inflammation grade, on the other, as demonstrated in our study, may suggest that mast cells account for fibrosis progression in patients with NAFLD together with other inflammatory cells from portal areas. However, the strong correlation between fibrosis grade and mast cells content, particularly as regards mast cells restricted to portal areas and fibrous septa, makes it highly probable that these cells play a central role in the pathogenesis of liver fibrosis in patients with nonalcoholic fatty liver disease.

\section{Conclusions}

In the study, we demonstrated a strong positive correlation between the number of mast cells located within portal areas and fibrous septa and liver fibrosis grade in patients with nonalcoholic fatty liver disease. The accumulation of mast cells in sites such as portal areas or fibrous septa contributes to fibrosis progression. This occurs because mast cells topically release various mediators that have an impact on their environment by attracting other inflammatory cells, inducing inflammation and regulating angiogenesis. Mast cells also stimulate HSCs and portal fibroblasts to produce ECM components, including collagen. The positive correlation between fibrosis grade and MC total count as well as an interdependence between fibrosis grade and portal inflammation grade suggest that portal mast cells and portal inflammatory cells contribute equally to fibrosis progression in patients with NAFLD. However, since the correlation between fibrosis grade and MC count is stronger, particularly with respect to those restricted to portal areas and fibrous septa, mast cells in the above locations may play a central role in the pathogenesis of liver fibrosis in patients with NAFLD. The lack of correlation between fibrosis grade and mast cell content within liver lobules indicates that MCs located away from portal areas and fibrous septa do not participate in fibrosis progression.

We would like to thank Mirostawa Korn for preparing tissues for histological examination.

The authors declare no conflict of interest.

\section{References}

1. Matteoni CA, Younossi ZM, Gramlich T, et al. Nonalcoholic fatty liver disease: a spectrum of clinical and pathological severity. Gastroenterology 1999; 116: 1413-1419.

2. Friedman SL. Mechanisms of hepatic fibrogenesis. Gastroenterology 2008; 134: 1655-1669.

3. Wells RG, Kruglov E, Dranoff JA. Autocrine release of TGF-beta by portal fibroblasts regulates cell growth. FEBS Lett 2004; 559: 107-110.

4. Kisseleva T, Uchinami H, Feirt N, et al. Bone marrow-derived fibrocytes participate in pathogenesis of liver fibrosis. J Hepatol 2006; 45: 429-438.

5. Schuppan D, Afdhal NH. Liver cirrhosis. Lancet 2008; 371 : 838-851.

6. Desai HG. Cryptogenic cirrhosis: a vanishing entity. J Assoc Physicians India 2009; 57: 251-255.

7. Wynn TA. Cellular and molecular mechanizms of fibrosis, J Pathol 2008; 214: 199-210.

8. Schuppan D. Structure of extracellular matrix in normal and fibrotic liver: collagens and glycoproteins. Semin Liver Dis 1990; 10: 1-10.

9. Reeves HL, Friedman SL. Activation of hepatic stellate cells: a key issue in liver fibrosis. Front Biosci 2002; 7: 808-826.

10. Ramadori G, Saile B. Portal tract fibrogenesis in the liver. Lab Invest 2004; 84: 153-159.

11. Forbes SJ, Russo FP, Rey V, et al. A significant proportion of myofibroblasts are of bone marrow origin in human liver fibrosis. Gastroenterology 2004; 126: 955-963.

12. Gressner AM, Weiskirchen R, Breitkopf $\mathrm{K}$, et al. Roles of TGF-beta in hepatic fibrosis. Front Biosci 2002; 7: 793807.

13. Metcalfe DD, Baram D, Mekori YA. Mast cells. Physiol Rev 1997; 77: 1033-1079.

14. Sawicki W, Malejczyk J. Tkanka łączna właściwa. In: Histologia. Sawicki W (ed.). $6^{\text {th }}$ ed. Wydawnictwo Lekarskie PZWL, Warszawa 2012; 147-173.

15. Kumar D, Pandya SK, Varshney S, et al. Temporal immunometabolic profiling of adipose tissue in HFD-induced obesity: manifestations of mast cells in fibrosis and senescence. Int J Obes (Lond) 2019; 43: 1281-1294.

16. Lin KQ, Zhu LB, Zhang XM, et al. Role of mast cells in estrogen-mediated experimental endometriosis in rats. Zhejiang Da Xue Xue Bao Yi Xue Ban 2015; 44: 269-277.

17. Zhu TH, Ding SJ, Li TT, et al. Estrogen is an important mediator of mast cell activation in ovarian endometriomas. Reproduction 2018; 155: 73-83.

18. Hirai S, Ohyane C, Kim YI, et al. Involvement of mast cells in adipose tissue fibrosis. Am J Physiol Endocrinol Metab 2014; 306: 247-255.

19. Kazama I, Baba A, Endo Y, et al. Mast cell involvement in the progression of peritoneal fibrosis in rats with chronic renal failure. Nephrology (Carlton) 2015; 20: 609-616.

20. Kong P, Christia P, Frangogiannis NG. The pathogenesis of cardiac fibrosis. Cell Mol Life Sci 2013; 71: 549-574.

21. El-Mohandes EM, Moustafa AM, Khalaf HA, et al. The role of mast cells and macrophages in amiodarone induced pulmonary fibrosis and the possible attenuating role of atorvastatin. Biotech Histochem 2017; 92: 467-480.

22. Armbrust A, Batusic D, Ringe B, et al. Mast cells distribution in human liver disease and experimental rat liver fibrosis. Indications for mast cell participation in development of liver fibrosis. J Hepatol 1997; 26: 1042-1054.

23. Farreil DJ, Hines JE, Walls AF, et al. Intrahepatic mast cells in chronic liver diseases. Hepatology 1995; 22: 1175-1181.

24. Akiyoshi H, Terada T: Mast cell, myofibroblast and nerve terminal complexes in carbon tertrachloride-induced cirrhotic rat livers. J Hepatol 1998; 29: 112-119. 
25. Yamashiro M, Kouda W, Kono N, et al. Distribution of intrahepatic mast cells in various hepatobiliary disorder. An immunohistochemical study. Virchows Arch 1998; 433: 471-479.

26. Sugihara A, Tsujimura T, Fujita Y, et al. Evaluation of role of mast cells in the development of liver fibrosis using mast cell-deficient rats and mice. J Hepatol 1999; 30: 859-867.

27. Bensley SH. Pinacyanol erythrosinate as a stain for mast cells. Stain Technol 1952; 27: 269-273.

28. Brunt EM, Kleiner DE, Wilson LA, et al. NASH Clinical Research Network: Portal Chronic Inflammation in Nonalcoholic Fatty Liver Disease (NAFLD): A Histologic Marker of Advanced NAFLD - Clinicopathologic Correlations from the Nonalcoholic Steatohepatitis Clinical Research Network. Hepatology 2009; 49: 809-820.

29. Kleiner DE, Brunt EM, Van Natta M, et al. Design and validation of a histological scoring system for nonalcoholic fatty liver disease. Hepatology 2005; 41: 1313-1321.

30. Basaranoglu M, Kayacetin S, Yilmaz N, et al. Understanding mechanisms of the pathogenesis of nonalcoholic fatty liver disease. World J Gastroenterol 2010; 16: 2223-2226.

31. Chavez-Tapia NN, Uribe M, Ponciano-Rodriguez G, et al. New insights into the pathophysiology of nonalcoholic fatty liver disease. Ann Hepatol 2009; 8: Suppl 1: 9-17.

32. Stoyanova II. Relevance of Mast cells and hepatic lobule innervation to liver injury. Rom J Gastroenterol 2004; 13: 203-209.

33. Liaskou E, Wilson DV, Oo YH. Innate Immune Cells in Liver Inflammation. Mediators Inflamm 2012; 2012: 949157.

34. Gruber BL, Kew RR, Jelaska A, et al. Human Mast cells activate fibroblasts: tryptase is a fibrogenic factor stimulating collagen messenger ribonucleic acid synthesis and fibroblasts chemotaxis. J immunol 1997; 158: 2310-2317.

35. Ruoss SJ, Hartmann T, Caughey GH. Mast cell tryptase is a mitogen for cultured fibroblasts. J Clin Invest 1991; 88: 493-499.

36. Hügle T. Beyond allergy: the role of mast cells in fibrosis. Swiss Med Wkly 2014; 144: w13999.

37. Elpek GÖ. Angiogenesis and liver fibrosis. World J Hepatol 2015; 7: 377-391.

38. Valfrè BL, Novo E, Cannito S, et al. Angiogenesis and liver fibrogenesis. Histol Histopathol 2009; 24: 1323-1341.

39. Groneberg DA, Bester C, Grützkau A, et al. Mast cells and vasculature in atopic dermatitis - potential stimulus of neoangiogenesis. Allergy 2005; 60: 90-97.

40. Puxeddu I, Ribatti D, Crivellato E, et al. Mast cells and eosinophils: a novel link between inflammation and angiogenesis in allergic diseases. J Allergy Clin Immunol 2005; 116: 531-536.

41. Crivellato E, Nico B, Ribatti D. Mast cell contribution to tumor angiogenesis: a clinical approach. Eur Cytokine Netw 2009; 20: 197-206.

42. Qu Z, Liebler JM, Powers MR, et al. Mast cells are a major source of basic fibroblast growth factor in chronic inflammation and cutaneous hemangioma. Am J Pathol 1995; 147 564-573.

43. Sörbo J, Jakobson A, Norrby K. Mast cell histamine is angiogenic through receptors for histamine 1 and histamine 2. Int J Exp Pathol 1994; 75: 43-50.

44. Ribatti D, Roncali L, Nico B, et al. Effects of exogenous heparin on the vasculogenesis of the chorioallantoic membrane. Acta Anat (Basel) 1987; 130: 257-263.

45. Kanbe N, Tanaka A, Kanbe M, et al. Human mast cells produce matrix metalloproteinase 9. Eur J Immunol 1999; 29: 2645-2649

46. Fernández M, Semela D, Bruix J, et al. Angiogenesis in liver disease. J Hepatol 2009; 50: 604-620.

47. Amarapurkar AD, Amarapurkar DN, Vibhav S, et al. Angiogenesis in chronic liver disease. Ann Hepatol 2007; 6: $170-173$.
48. Ciupińska-Kajor M, Hartleb M, Kajor M, et al. Hepatic angiogenesis and fibrosis are common features in morbidly obese patients. Hepatol Int 2013; 7: 233-240.

49. Schluger LK, Sheiner PA, Thung SN, et al. Severe recurrent cholestatic hepatitis $\mathrm{C}$ following orthotopic liver transplantation. Hepatology 1996; 23: 971-976.

50. Bonnard P, Lescure FX, Amiel C, et al. Documented rapid course of hepatic fibrosis between two biopsies in patients coinfected by HIV and HCV despite high CD4 cell count. J Viral Hepat 2007; 14: 806-811.

51. Charlton M, Krishnan A, Viker K, et al. Fast food diet Mouse: novel small animal model of $\mathrm{NASH}$ with ballooning, progressive fibrosis, and high physiological fidelity to the human condition. Am J Physiol Gastrointest Liver Physiol 2011; 301: 825-834.

52. Czaja AJ, Carpenter HA. Progressive fibrosis during corticosteroid therapy of autoimmune hepatitis. Hepatology 2004; 39: 1631-1638.

53. Czaja AJ. Hepatic inflammation and progressive liver fibrosis in chronic liver disease. World J Gastroenterol 2014; 20: 2515-2532.

54. Colombat M, Charlotte F, Ratziu V, et al. Portal lymphcytic infiltrate in alcoholic liver disease. Hum Pathol 2002; 33: 1170-1174

55. Liew PL, Lee WJ, Lee YC, et al. Hepatic histopathology of morbid obesity: concurence of Rother forms of chronic liver disease. Obes Surg 2006; 16: 1584-1593.

\section{Address for correspondence}

Ewa Lewandowska

Department of Histology and Embryology

Interfaculty Chair of Anatomy and Histology

Medical University of Lodz

Zeligowskiego 7/9

90-752 Lodz, Poland

Tel. +484227252 65, +48422725267

e-mail: ewa.lewandowska@umed.lodz.pl 